\title{
Fractional-calculus-based control scheme for dynamical systems with input uncertainty
}

\author{
Mohammad Pourmahmood Aghababa ${ }^{1 *}$
}

\section{"Correspondence:}

m.p.aghababa@ee.uut.ac.ir

m.p.aghababa@gmail.com

${ }^{1}$ Faculty of Electrical Engineering,

Urmia University of Technology,

Urmia, Iran

\section{照 Springer}

\begin{abstract}
Since the existence of unwanted oscillations should be avoided in practical systems, this article investigates active vibration and oscillation suppression of two-degree-of-freedom dynamical systems using a novel variable structure control methodology. Owing to high stability and generality of the fractional-calculus-based differential equations, a non-integer-order sliding surface is proposed. Afterward, the occurrence of the sliding motion is ensured using a switching control rule. The effects of the input nonlinearities, which are usually existed in mechanical actuators, are fully dealt with using the introduced fractional sliding modes. In addition, unknown lumped uncertainties are considered to disturb the system dynamics. As a result, the proposed controller is robust against system and control fluctuations and can handle bounded external perturbations. Moreover, careful stability synthesis is developed to theoretically confirm the control designs. Finally, two numerical case studies, which include oscillation control of a magnetic bearing system and a gyroscope device, are provided to demonstrate the superior performance of the suggested control technology.
\end{abstract}

Keywords: Fractional sliding surface; Oscillation elimination; Input nonlinearity; External perturbation; Active control

\section{Introduction}

It is well known that, in many cases, when a physical system is excited by some external forces, it starts to oscillate with finite amplitude and frequency [1, 2]. For example, consider a mechanical system intrinsic stresses concentrated by oscillation. Such condition not only can result strain fractures of the system's mechanical parts but also decreases machine power, engenders noise and disturbance and enlarges friction. Generally speaking, any form of unwanted oscillation may yield to machine performance degradation, more energy spending, noise generation, reliability and efficiency decrease, damaging mechanical parts of the system and human discomfort [3]. Therefore, some alternatives should be adopted to compensate and suppress unnecessary oscillations.

Generally speaking, passive, semi-active and active control methods are the main control methodologies for oscillation suppressor design [3]. For system steady state response manipulation, the passive oscillation control adopts some mechanical elements, such as springs and dampers, to add absorbers, isolators, suspensions or resonators to the system. When the oscillation frequency exceeds some threshold values, semi-active approaches with adaptive springs and dampers are usually implemented. However, many real world

(c) The Author(s) 2019. This article is distributed under the terms of the Creative Commons Attribution 4.0 International License (http://creativecommons.org/licenses/by/4.0/), which permits unrestricted use, distribution, and reproduction in any medium, provided you give appropriate credit to the original author(s) and the source, provide a link to the Creative Commons license, and indicate if changes were made. 
applications have low frequency oscillations. In this case, active oscillation control is realized using external actuators [4-6].

The foundation of fractional calculus has been established by Leibniz and L'Hospital in 1965 [7]. Fractional-calculus-based differential equations are more general mathematic tools compared to the conventional integer-order differentials in which they adopt a realvalued derivative (or integration) operator instead of just an integer-valued differentiator (or integrator). However, the fractional calculus was not successful to find useful engineering applications for several centuries and the progresses of this area were quite slow. Nevertheless, in the recent decades, the theory of fractional derivatives and integrals has been utilized for precisely description and modeling of a wide range of real-world phenomena observed in practical systems and situations.

Owing to the limited operation of control actuators, such as electronic circuits composed of operational amplifiers, electromechanical apparatus, pneumatic devices and hydraulic machines, there are usually a number of nonlinearities in control inputs which include backslash, hysteresis, saturation, dead-zone, etc. The existence of such nonlinearities in the controller can generate irregular behaviors in the system outputs, decrease the control performance, bias extra oscillations in the system response and even result in system failure as well as unstable closed-loop systems. Hence, the influence of the input nonlinearity should not be ignored in the procedure of analysis, design and implementation of active control systems. In addition, since functional systems are inevitably perturbed by parameter fluctuations and external perturbations, realization of a robust oscillation controller is essential to evade degradation of the arranged performance for the system outputs. In [6], stability synthesis of fuzzy control systems in spite of sector input nonlinearities has been studied. Orszulik and Shan [8] have considered the Lyapunov-based classical feedback control of piezoelectric devices with the hysteresis nonlinearity. In the work [9], sliding-mode variable structure controllers have been derived for attitude control of underwater vehicles and delayed oscillatory systems with considering input nonlinearities. An artificial neural network control algorithm has been proposed in [10] for robotic manipulators with actuator saturation nonlinearity. A PID-iterative learning control approach for tackling the actuator nonlinearity has been designed by Huang and Chen for a biomedical device [11]. Finite-time stability synthesis of special nonlinear systems in spite of the input nonlinearity has been addressed in [12]. Control design of non-integer-order systems in the presence of nonlinear inputs has been also reported in the literature [13, $14]$.

Motivated by the aforementioned statements, the problem of robust oscillation attenuation of uncertain two-dimensional nonlinear dynamical systems subjected to input nonlinearity is investigated in this paper. Since the sliding modes are robust effective control strategies adopted for applied dynamical systems in the literature [15-20], to enhance the controller stability, a hybrid fractional-integer-order sliding-mode control methodology is introduced. As a result, unknown system parameter variations and external disturbances as well as both linear and nonlinear input controls are fully taken into account and efficient switching control rules are developed to derive the system trajectories to the arranged non-integer-order sliding surface. At last, computer simulations are presented for two mechanical devices.

This article is structured as follows: Sect. 2 gives preliminaries for fractional-calculus theory. Section 3 stands for system demonstration and problem statement. Section 4 pro- 
vides the introduced non-integer-order sliding-mode controllers. In Sect. 5, two illustrative numerical case studies are included. Finally, conclusions are provided in Sect. 6.

\section{Fractional-calculus preliminaries}

In this section, some basics of fractional-calculus theory as well as fractional stability theorems are presented.

Definition 1 ([7]) The fractional integral of a function $f(t)$ is defined as follows:

$$
{ }_{t_{0}} I_{t}^{\alpha} f(t)=\frac{1}{\Gamma(\alpha)} \int_{t_{0}}^{t} \frac{f(\tau)}{(t-\tau)^{1-\alpha}} d \tau
$$

in which $t_{0}$ stands for the starting time, $\alpha \in R^{+}$represents the order of integration and $\Gamma(\cdot)$ shows the Gamma function defined as follows:

$$
\Gamma(z)=\int_{t_{0}}^{\infty} t^{z-1} e^{-t} d t
$$

Definition 2 ([7]) For the function $f(t)$, the Caputo derivative is presented by

$$
{ }_{t_{0}}^{C} D_{t}^{\alpha} f(t)={ }_{t_{0}} I_{t}^{(m-\alpha)} \frac{d^{m}}{d t^{m}} f(t)=\frac{1}{\Gamma(m-\alpha)} \int_{t_{0}}^{t} \frac{f^{(m)}(\tau)}{(t-\tau)^{\alpha-m+1}} d \tau
$$

in which $m-1<\alpha<m \in N$.

Property 1 ([7]) For the Caputo derivatives in the case of $m=1$, one has

$$
{ }_{t_{0}}^{C} D_{t t_{0}}^{\alpha} I_{t}^{\alpha} f(t)={ }_{t_{0}}^{\mathrm{RL}} D_{t}^{\alpha} f(t) t_{t_{0}} I_{t}^{\alpha} f(t)=f(t) .
$$

Property 2 ([7]) First-order integration of the Caputo derivatives is as follows:

$$
{ }_{t_{0}} I_{t t_{0}}^{\alpha C} D_{t}^{\alpha} f(t)=f(t)-f\left(t_{0}\right)
$$

Remark 1 Through this article, the Caputo definition is denoted via $D^{\alpha}$.

In what follows, the chief stability theories for fractional-order systems are restated.

Definition 3 ([21]) The constant $x_{0}$ is an equilibrium point of fractional system $D^{\alpha} x(t)=$ $f(x, t)$, if and only if $f(x, t)=D^{\alpha} x_{0}$.

Theorem 1 ([22]) For $|\arg (\operatorname{eig} A)|>\alpha \pi / 2$, zero is the asymptotic stable unique equilibrium point of (6).

$$
D^{\alpha} x=A x, \quad x(0)=x_{0}
$$

in which $1<\alpha<2, x \in R^{n}$ and $A \in R^{n \times n}$. 
Definition 4 ([21]) The solution of $D^{\alpha} x(t)=f(x, t)$ is said to be Mittag-Leffler (asymptotic) stable if the following condition holds:

$$
\|x(t)\| \leq\left\{m\left[x\left(t_{0}\right)\right] E_{\alpha}\left(-\lambda\left(t-t_{0}\right)^{\alpha}\right)\right\}^{b}
$$

where $E_{\alpha}(z)=\sum_{k=0}^{\infty} \frac{z^{k}}{\Gamma(k \alpha+1)}$ is the Mittag-Leffler function, $\lambda, b>0, m(0)=0, m(z) \geq 0$ and $m(z)$ is locally Lipschitz function.

Theorem 2 ([21]) For $\alpha \in(0,1]$, if

(i) the origin is an equilibrium point of the fractional system

$$
D^{\alpha} x(t)=f(x, t)
$$

(ii) $f(x, t)$ has a Lipschitz constant $l>0$ and

(iii) there exists a function $V(t, x(t))$ and a class-K functions $\alpha_{i}, i=1,2,3$ with

$$
\alpha_{1}(\|x\|) \leq V(t, x) \leq \alpha_{2}(\|x\|), \quad D^{\alpha} V(t, x) \leq-\alpha_{3}(\|x\|)
$$

then zero is the asymptotic stable equilibrium point of the system (8).

Lemma 1 ([23]) For $\alpha \in(0,1]$, the following formula is valid for the Caputo derivatives:

$$
\frac{1}{2}{ }^{t_{0}} D_{t}^{\alpha} f^{2}(t) \leq f(t)_{t_{0}}^{C} D_{t}^{\alpha} f(t)
$$

\section{System description and problem statement}

To analysis and synthesis the oscillation of a dynamical system, the first step is to provide a model for the system behavior description using mathematical formulas based on the physics and nature of the system. In this regard, most of complicated two-degree-offreedom (2DoF) mechanical systems, such as micro-mechanical resonators, gyroscopes, robot manipulators, piezoelectric actuators, horizontal platforms, Duffing oscillators, 2DoF structures, inverted pendulums and magnetic bearings, can be effectively characterized by the following non-autonomous second-order dynamical equations:

$$
\ddot{x}=F(X, t)+\Delta f(X, t)+d(X, t)+(g(X, t)+\Delta g(X, t)) \varphi(u(t))
$$

in which $X=[x, \dot{x}]^{T} \in R^{2}$ is the system state vector, $F(X, t)$ is a nonlinear function, $\Delta f(X, t)$ represents system linear and/or nonlinear uncertainties representing modeling errors, parameter variations and un-modeled dynamics, $d(X, t)$ shows an external perturbation, $g(X, t)>0$ is an invertible control gain function, $\Delta g(X, t)$ represents control uncertainties, $\varphi(\cdot)$ is a linear or nonlinear function introduced later and $u(t) \in R$ is the single control signal.

Defining $x_{1}=x$ and $x_{2}=\dot{x}$, one can rewrite the model (11) as follows:

$$
\begin{aligned}
& \dot{x}_{1}=x_{2}, \\
& \dot{x}_{2}=f(X, t)+\Delta L(X, u, t)+g(X, t) \varphi(u(t)),
\end{aligned}
$$


where $f(X, t)$ is a continuous nonlinear function and $\Delta L(X, u, t)=\Delta f(X, t)+d(X, t)+$ $\Delta g(X, t) \varphi(u(t))$ is named the system lumped uncertainly.

Assumption 1 The system lumped uncertainty is considered to be bounded via

$$
|\Delta L(X, u, t)| \leq a\|X\|+b\|u\|+c
$$

in which $a, b, c<\infty$ are known positive constants.

Remark 2 It is noted that the exact value of the lumped uncertainty $\Delta L(X, u, t)$ is generally unknown. However, in real applications, one can introduce some reasonable fixed upper bounds for the system uncertainties and external disturbances. On the other hand, in Assumption 1, we use two dynamic bounds (i.e. $a\|X\|$ and $b\|u\|$ ) along a constant bound (i.e. $c$ ) to tackle the lumped uncertainty $\Delta L(X, u, t)$ as a function of the system states and control inputs. The utilization of these dynamic terms in the control inputs can aid the controller to overcome the lumped uncertainties in a more reliable manner rather than applying classic fixed bounds which are common in the traditional control signals.

Control objective: The main aim of this article is to derive a nonlinear non-integer-order robust sliding-mode controller in order to suppress the vibratory and oscillatory behavior of the uncertain system (12) even if some input nonlinearities are present in the actuators.

\section{Design of fractional sliding active control scheme}

Among available active control methodologies, the sliding-mode strategy is the most common method in oscillation attenuation, because it can overcome unavoidable parameter fluctuations and external perturbations which degrade the system performance. Moreover, the sliding-mode technology has simple structure and high stability features. Thus, this article applies a new fractional sliding mode for oscillation suppression of practical integer-order systems. The design steps of the proposed sliding-mode controller are given below.

Here, a non-integer-order sliding manifold is designed as

$$
s(t)=D^{-\alpha} \ddot{x}(t)+\lambda x(t)
$$

where $\lambda>0$ is a constant.

Now, the fractional-order dynamics of the sliding manifold (14) can be achieved as follows:

$$
s(t)=0 \quad \rightarrow \quad D^{-\alpha} \ddot{x}(t)=D^{-\alpha+2} x(t)=-\lambda x(t) .
$$

Theorem 3 Zero is the asymptotic stable equilibrium point of the dynamics (15).

Proof Referring to Theorem 1, the stability condition for the linear system (15) is $|\arg (\lambda)|>$ $(2-\alpha) \pi / 2$. Since the eigenvalue of the non-integer-order sliding manifold dynamics (15) is equal to $-\lambda$, one can conclude that $0<\alpha<1$ (or $1<(2-\alpha)<2$ ) meets the stability criterion $|\arg (\lambda)|=\pi>(2-\alpha) \pi / 2)$. Hence, the stability circumvent of Theorem 1 is met and the sliding-mode dynamics (15) is asymptotically stable. So, the proof is ended. 
After designing a desired sliding manifold, the next step is to propose a control law to ensure the occurrence of the sliding motion. To derive a sliding-mode control law, first the case of linear input is considered. So, the function $\varphi(\cdot)$ is removed from (12) and the resulting system is changed into the following structure:

$$
\begin{aligned}
& \dot{x}_{1}=x_{2}, \\
& \dot{x}_{2}=f(X, t)+\Delta L(X, u, t)+g(X, t) u(t) .
\end{aligned}
$$

The proper fractional-order sliding control law is provided as

$$
\left.u(t)=-g^{-1}(X, t)\left(f(X, t)+\lambda D^{\alpha} x_{1}(t)+\left(K_{1}\|X\|+K_{2}\|u\|+K_{3}\right) \operatorname{sgn}(s)\right)\right)
$$

in which $K_{1}>a, K_{2}>b$ and $K_{3}>c$ are three constants.

Theorem 4 The control signal (17) is able to reach the system state trajectories (16) with the condition (14) to the sliding manifold $s(t)=0$.

Proof One can select a Lyapunov function candidate as

$$
V_{1}(t)=0.5 s^{2}
$$

Taking the fractional-order derivative of $V(t)$ and based on Lemma 1, one has

$$
D^{\alpha} V_{1} \leq s D^{\alpha} s .
$$

Using the proposed sliding surface (14), we have

$$
D^{\alpha} V_{1} \leq s D^{\alpha}\left(D^{-\alpha} \ddot{x}(t)+\lambda x(t)\right) .
$$

Based on $\ddot{x}(t)=f(X, t)+\Delta L(X, u, t)+g(X, t) u(t)$ and Property 1 , one obtains

$$
D^{\alpha} V_{1} \leq s\left(f(X, t)+\Delta L(X, u, t)+g(X, t) u(t)+\lambda D^{\alpha} x(t)\right) .
$$

It is clear that

$$
D^{\alpha} V_{1} \leq s\left(f(X, t)+g(X, t) u(t)+\lambda D^{\alpha} x(t)\right)+|s||\Delta L(X, u, t)| .
$$

Referring to Assumption 1 and the inequality (13), one gets

$$
D^{\alpha} V_{1} \leq s\left(f(X, t)+g(X, t) u(t)+\lambda D^{\alpha} x(t)\right)+|s|(a\|X\|+b\|u\|+c) .
$$

Substituting $u(t)$ from (17) into (23), it yields

$$
\begin{aligned}
D^{\alpha} V_{1} & \leq-|s|\left(K_{1}\|X\|+K_{2}\|u\|+K_{3}\right)+|s|(a\|X\|+b\|u\|+c) \\
& =|s|\left(\left(a-K_{1}\right)\|X\|+\left(b-K_{2}\right)\|u\|+c-K_{3}\right) \leq-\left(K_{3}-c\right)|s| \leq 0 .
\end{aligned}
$$




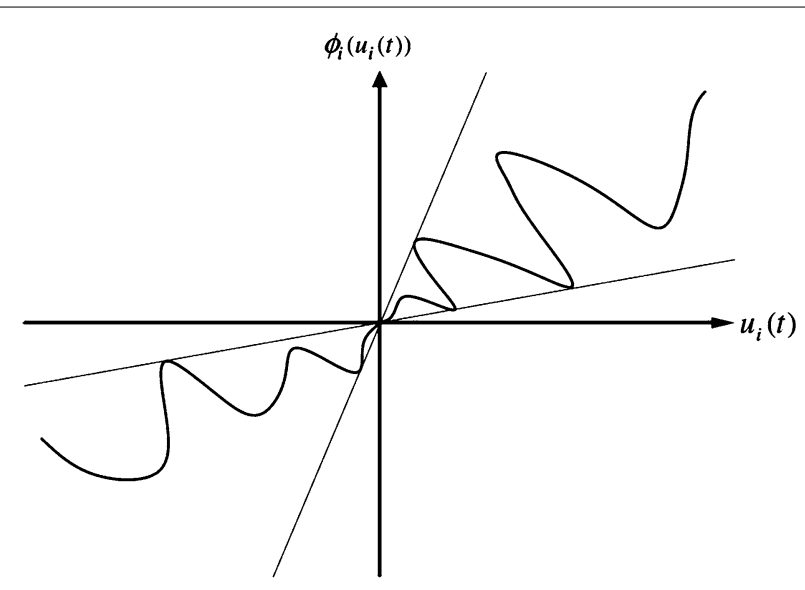

Figure 1 A schematic diagram of a typical input nonlinearity

Consequently, based on Theorem 2, the system states will converge to $s(t)=0$. Thus, the proof is completed.

Now, assume that the control actuator is subjected to sector nonlinearities. In this case, we modify the proposed sliding-mode technology (17) so that the existence of the reaching phase is ensured. A schematic diagram of a typical input nonlinearity is displayed in Fig. 1.

In (12), it is assumed that $\varphi(u(t))$ with $\varphi(0)=0$ is inside the sector $[\rho, \mu], \rho>0$ as follows:

$$
\rho u^{2} \leq u \varphi(u) \leq \mu u^{2} .
$$

To tackle the nonlinear phenomenon of the control input, a robust sliding control rule is proposed by

$$
u(t)=-\frac{\operatorname{sgn}(s)}{\rho g(X, t)}\left(|f(X, t)|+\lambda\left|D^{\alpha} x_{1}(t)\right|+K_{1}\|X\|+K_{2}\|u\|+K_{3}\right)=-\xi \operatorname{sgn}(s)
$$

with $\xi=\frac{1}{\rho g(X, t)}\left(|f(X, t)|+\lambda\left|D^{\alpha} x_{1}(t)\right|+K_{1}\|X\|+K_{2}\|u\|+K_{3}\right)>0$.

Remark 3 Substituting $u(t)$ in (26) by $u(t)=-\xi \operatorname{sgn}(s), \xi>0$, one obtains $\rho \xi^{2} \operatorname{sgn}(s)^{2} \leq$ $-\xi \operatorname{sgn}(s) \varphi(u) \rightarrow \rho \xi^{2} \leq-\xi \operatorname{sgn}(s) \varphi(u)$, multiplying $-|s|$ to both sides of the last inequality, one gets

$$
s \varphi(u) \leq-\rho \xi|s|
$$

The property is left to be adopted in the proof of Theorem 5 .

Theorem 5 The control rule (26) can reach the state trajectories of the system (12) with the conditions (13) and (25) to the sliding manifold $s(t)=0$.

Proof On the basis of the fractional stability theorem, a positive definite function is taken as

$$
V_{2}(t)=0.5 s^{2}
$$


Taking the fractional-order derivative of $V_{2}(t)$ and based on Lemma 1, one gets

$$
D^{\alpha} V_{2} \leq s D^{\alpha} s
$$

Using the proposed sliding surface (14), one can obtain

$$
D^{\alpha} V_{2} \leq s D^{\alpha}\left(D^{-\alpha} \ddot{x}(t)+\lambda x(t)\right) .
$$

Based on system dynamics (12) and Property 1, one has

$$
D^{\alpha} V_{2} \leq s\left(f(X, t)+\Delta L(X, u, t)+g(X, t) \varphi(u(t))+\lambda D^{\alpha} x(t)\right) .
$$

It is obvious that

$$
D^{\alpha} V_{2} \leq|s|\left(|f(X, t)|+\left|\lambda D^{\alpha} x(t)\right|+|\Delta L(X, u, t)|\right)+\operatorname{sg}(X, t) \varphi(u(t)) .
$$

Referring to Assumption 1 and the inequality (13), one can obtain

$$
D^{\alpha} V_{2} \leq|s|\left(|f(X, t)|+\left|\lambda D^{\alpha} x(t)\right|+a\|X\|+b\|u\|+c\right)+\operatorname{sg}(X, t) \varphi(u(t)) .
$$

Using Remark 3 and the inequality (27), we have

$$
D^{\alpha} V_{2} \leq|s|\left(|f(X, t)|+\left|\lambda D^{\alpha} x(t)\right|+a\|X\|+b\|u\|+c\right)-g(X, t) \rho \xi|s| .
$$

Substituting $\xi$ from (26) into (34), it yields

$$
\begin{aligned}
D^{\alpha} V_{1} & \leq-|s|\left(K_{1}\|X\|+K_{2}\|u\|+K_{3}\right)+|s|(a\|X\|+b\|u\|+c) \\
& =|s|\left(\left(a-K_{1}\right)\|X\|+\left(b-K_{2}\right)\|u\|+c-K_{3}\right) \leq-\left(K_{3}-c\right)|s| \leq 0 .
\end{aligned}
$$

Therefore, on the basis of Theorem 2, the system states will reach to $s(t)=0$. Hence, the proof is ended.

Remark 4 To prevent the occurrence of undesirable oscillations named chattering, which appear due to the existence of the $\operatorname{sgn}(s)$ function in the control inputs (17) and (26), we replace it by the continuous function $\tanh (\varepsilon s)$ with $\varepsilon>0$ a constant.

\section{Computer simulations}

In this section, two illustrative examples are included to emphasize the effectiveness and applicability of the introduced active control methodology.

\subsection{Magnetic bearing system}

A magnetic bearing system (see Fig. 2) is a mechanical device which benefits magnetic forces to swing a rotor shaft in midair. The useful applications of mechanical magnetic bearings in the fields of vacuum pumps, gas turbines, electric power apparatus, reaction wheels and jet engines have been reported in the literature. 


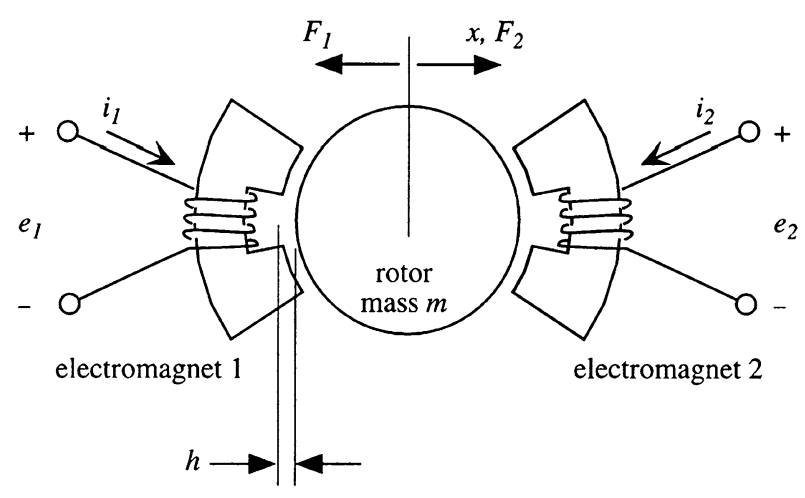

Figure 2 A magnetic bearing system [24]

A simplified two-dimensional motion equation of the magnetic bearing device is governed by

$$
m \ddot{x}(t)+c \dot{x}+k_{x} x+k_{i} i_{x}=0
$$

where $m=2.565$ denotes the rotor mass, $c=0.001$ shows the friction and $k_{x}=25.2$ and $k_{i}=40$ represent the position and current stiffness parameters, respectively.

Defining $x_{1}=x, x_{2}=\dot{x}$ and $u(t)=i_{x}$, the model (36) with a lumped uncertainty is rewritten as follows:

$$
\begin{aligned}
& \dot{x}_{1}=x_{2}, \\
& \dot{x}_{2}=\frac{c}{m} x_{2}+\frac{k_{x}}{m} x_{1}+\frac{k_{i}}{m} u(t)+\Delta L(X, u, t),
\end{aligned}
$$

where $\Delta L(X, u, t)=0.3 \sin \left(5 x_{2} x_{1}\right)+0.2 \tanh (3 t)$ is considered as the lumped uncertainties.

Referring to Eqs. (14) and (17), the sliding-mode controller is derived as follows:

$$
\begin{aligned}
& s(t)=D^{-0.1} \ddot{x}(t)+2 x(t), \\
& u(t)=-\frac{m}{k_{i}}\left(\frac{c}{m} x_{2}+\frac{k_{x}}{m} x_{1}+2 D^{0.1} x_{1}(t)+(0.4\|X\|+0.3) \tanh (50 s)\right) .
\end{aligned}
$$

The system is simulated with the starting points of $x_{1}(0)=-1$ and $x_{2}(0)=1$. Figure 3 depicts the state evolutions of the controlled magnetic bearing system. It is seen that there is no oscillation and the convergence to the origin is achieved as quickly as possible. For comparison, the state trajectory results of the fractional-order and integer-order terminal sliding modes given in [25] for the magnetic bearing system without input nonlinearities are illustrated in Fig. 4. One can see that the convergence times of the methods in [25] are about $5 \mathrm{sec}$. However, the introduced technique in this article achieves the convergence by $1 \mathrm{sec}$. This means that the proposed control strategy in this paper is faster and more robust against the lumped uncertainties than the available fractional and integer terminal sliding modes in the literature. The time evolution of the applied sliding manifold (38) is shown in Fig. 5. It is seen that the sliding motion occurs quickly. The time evolution of the control signal is displayed in Fig. 6. The time response of the applied control input in Ref. [25] 


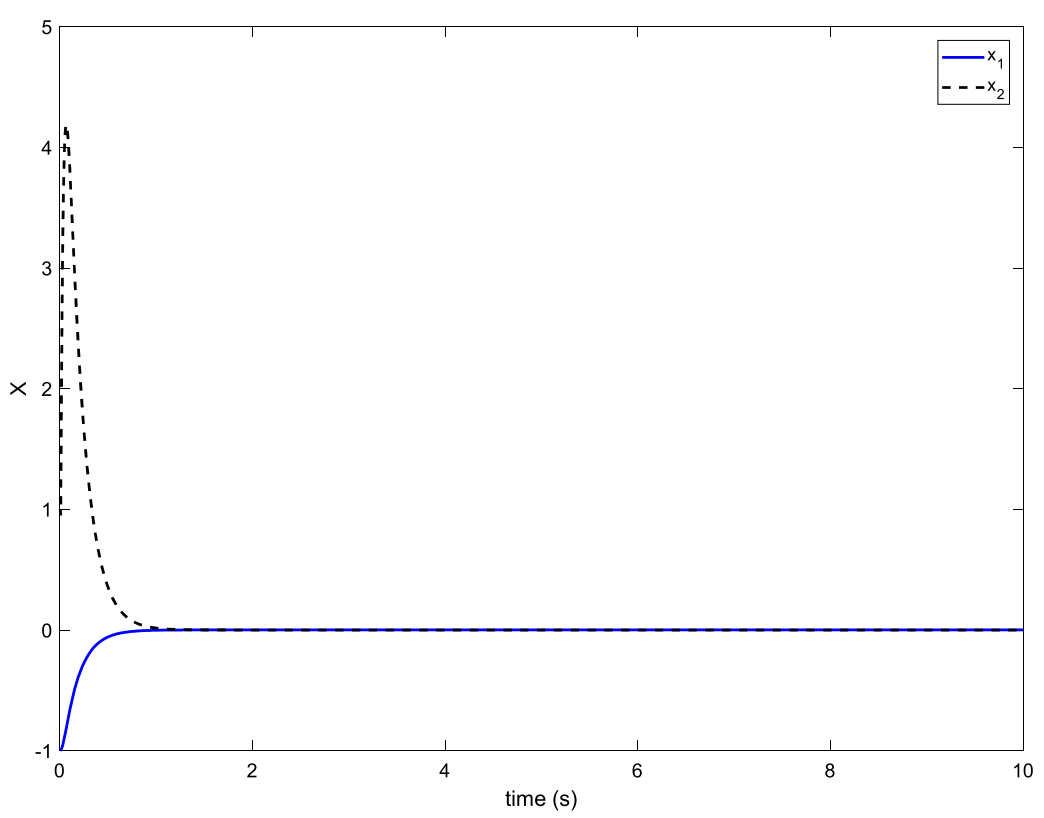

Figure 3 State evolutions of the magnetic bearing system obtained by the proposed method
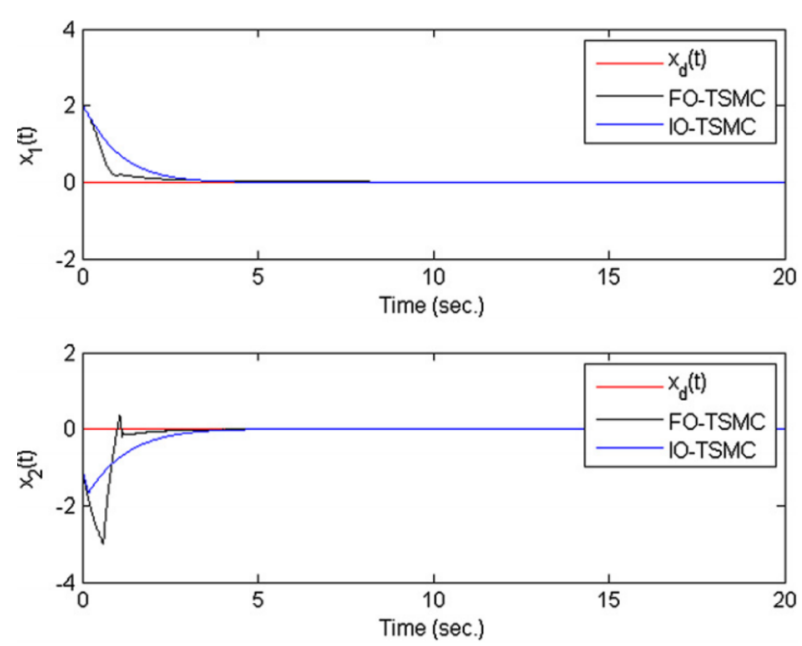

Figure 4 State evolutions of the magnetic bearing system obtained in [25]

is depicted in Fig. 7. Obviously, there are high frequency oscillations (chattering) on the control input proposed by the work [25] which limits the applicability of the corresponding controller in practice. Also, since the control signal of the work [25] provided in Fig. 7 does not converge to zero, the integral control effort will be infinite, avoiding the need of implementing the controller in practical situations. On the other hand, the amplitude of the control input developed in this research is finite with no harmful chattering and it converges to zero, implying that the proposed oscillation suppressor can be realized in practical situations. 


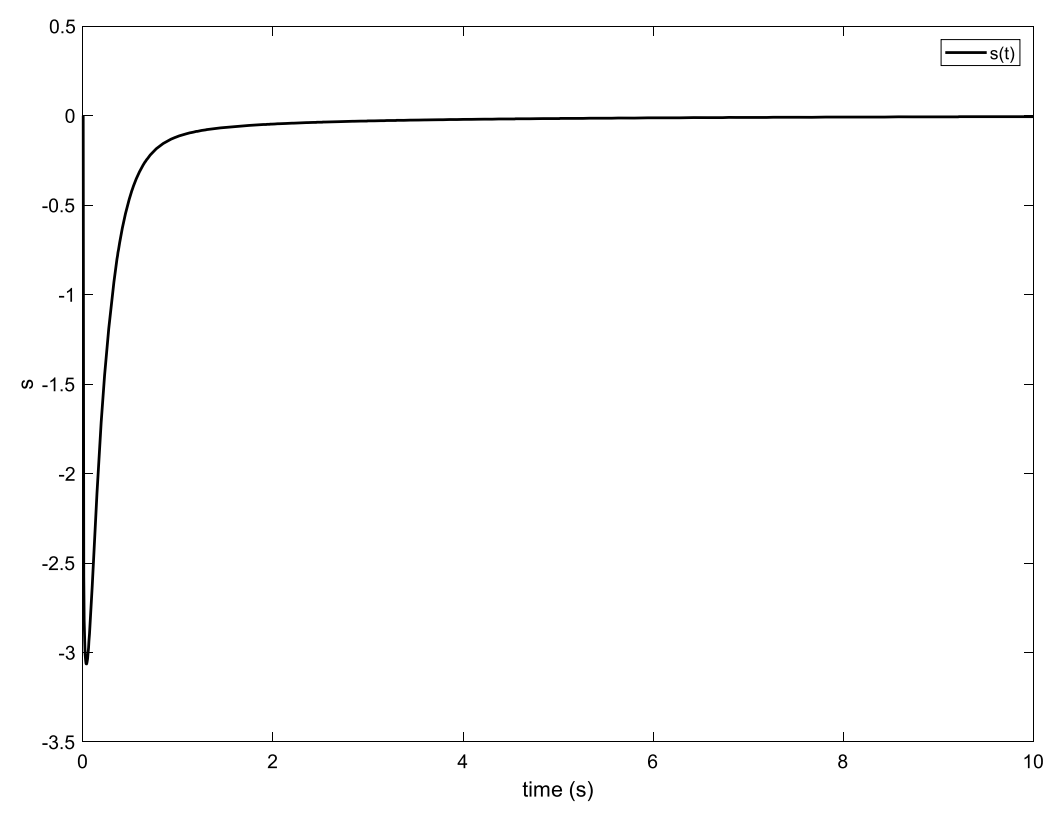

Figure 5 Time evolution of the applied sliding manifold (38)

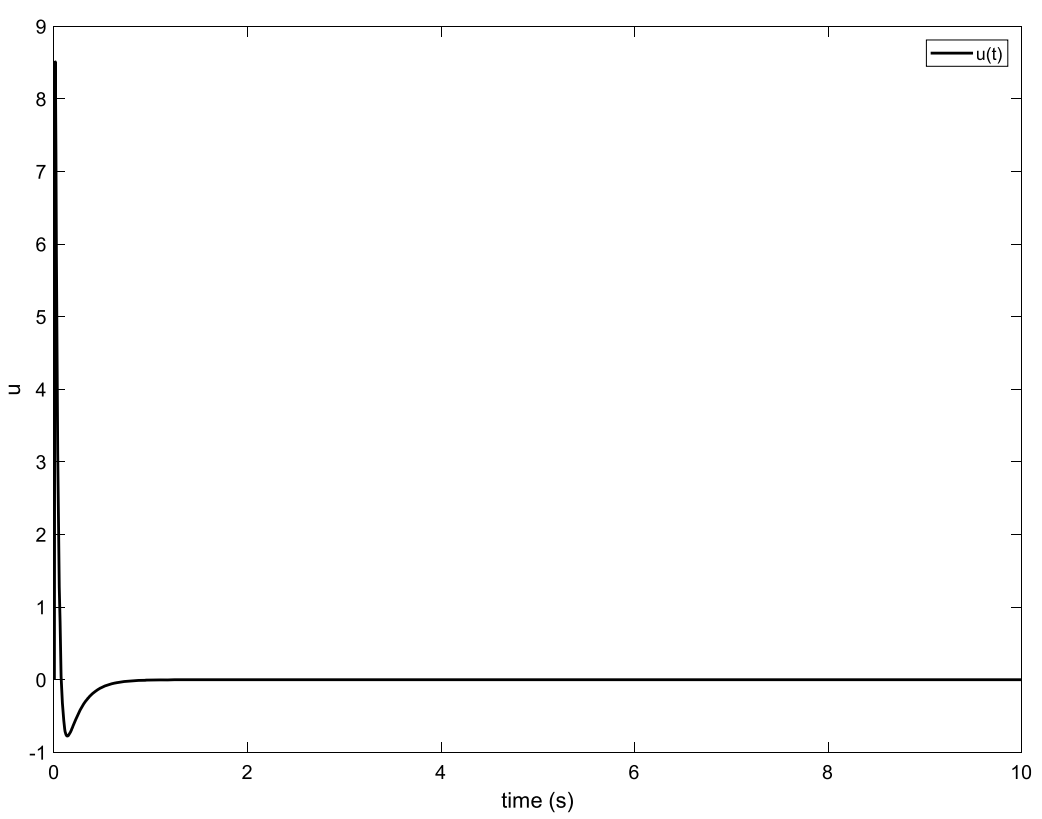

Figure 6 Time evolution of the control input (39) obtained by the proposed method

\subsection{Gyroscope system}

In this case, the gyroscope system which has valuable applications in many engineering fields such as navigation, mechatronics, optical device circuits, aeronautics applications and space engineering is adopted for the simulation purpose. The dynamical equations of 


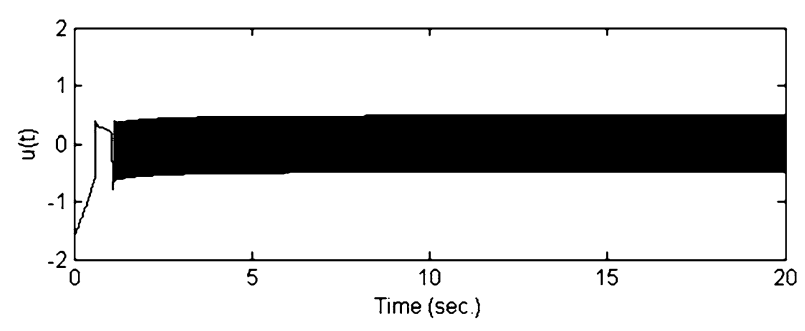

Figure 7 Time evolution of the control input (48) obtained in [25]

the gyroscope are given by

$$
\ddot{x}=\alpha^{2} \frac{\left(1-\cos x_{1}\right)^{2}}{\sin ^{3} x_{1}}+c_{1} x_{2}+c_{2} x_{2}^{3}-\beta \sin x_{1}-f \sin \omega t \sin x_{1} .
$$

Defining $x_{1}=x$ and $x_{2}=\dot{x}$ and adding a lumped uncertainty to the system dynamics, one can rewrite the gyroscope model (40) with a nonlinear input as follows:

$$
\begin{aligned}
\dot{x}_{1}= & x_{2}, \\
\dot{x}_{2}= & -\alpha^{2} \frac{\left(1-\cos x_{1}\right)^{2}}{\sin ^{3} x_{1}}-c_{1} x_{2}-c_{2} x_{2}^{3}+\beta \sin x_{1} \\
& +f \sin \omega t \sin x_{1}+\Delta L(X, u, t)+\varphi(u(t))
\end{aligned}
$$

in which $x_{1}$ represents the rotation angle, $x_{2}$ shows the rotation angle velocity, parametric and the base excitation is represented by $f \sin \omega t, c_{1} x_{2}$ and $c_{2} x_{2}^{3}$ are linear and nonlinear damping terms, respectively, and $\alpha^{2}=100, \beta=1, c_{1}=0.5, c_{2}=0.05, \omega=25$ and $f=35.5$ are constant system parameters.

It is well known that the gyroscope system has an oscillatory behavior and for the given parameter values this system displays chaotic behavior. The aim of this example is to suppress the oscillations of the uncertain gyroscope with input nonlinearity. The lumped uncertainty and input nonlinearity are supposed to be in the following forms, respectively:

$$
\begin{aligned}
& \Delta L(X, u, t)=0.2 \cos (2 t) x_{2}+0.1 \tanh (4 t) u(t)+0.4 \sin (t), \\
& \varphi(u(t))=(4+\sin (t)) u(t) .
\end{aligned}
$$

The vector $X(0)=[1,2]^{T}$ is taken as the initial conditions of the gyroscope system. And, according to Eq. (14), the following sliding manifold is established:

$$
s(t)=D^{-0.1} \ddot{x}(t)+x(t) .
$$

Subsequently, a sliding control rule is derived as

$$
\begin{aligned}
u(t)= & -\frac{\tanh (50 s)}{3}\left(\left|-\alpha^{2} \frac{\left(1-\cos x_{1}\right)^{2}}{\sin ^{3} x_{1}}-c_{1} x_{2}-c_{2} x_{2}^{3}+\beta \sin x_{1}+f \sin \omega t \sin x_{1}\right|\right. \\
& \left.+\left|D^{0.1} x_{1}(t)\right|+0.3\|X\|+0.2\|u\|+0.5\right) .
\end{aligned}
$$




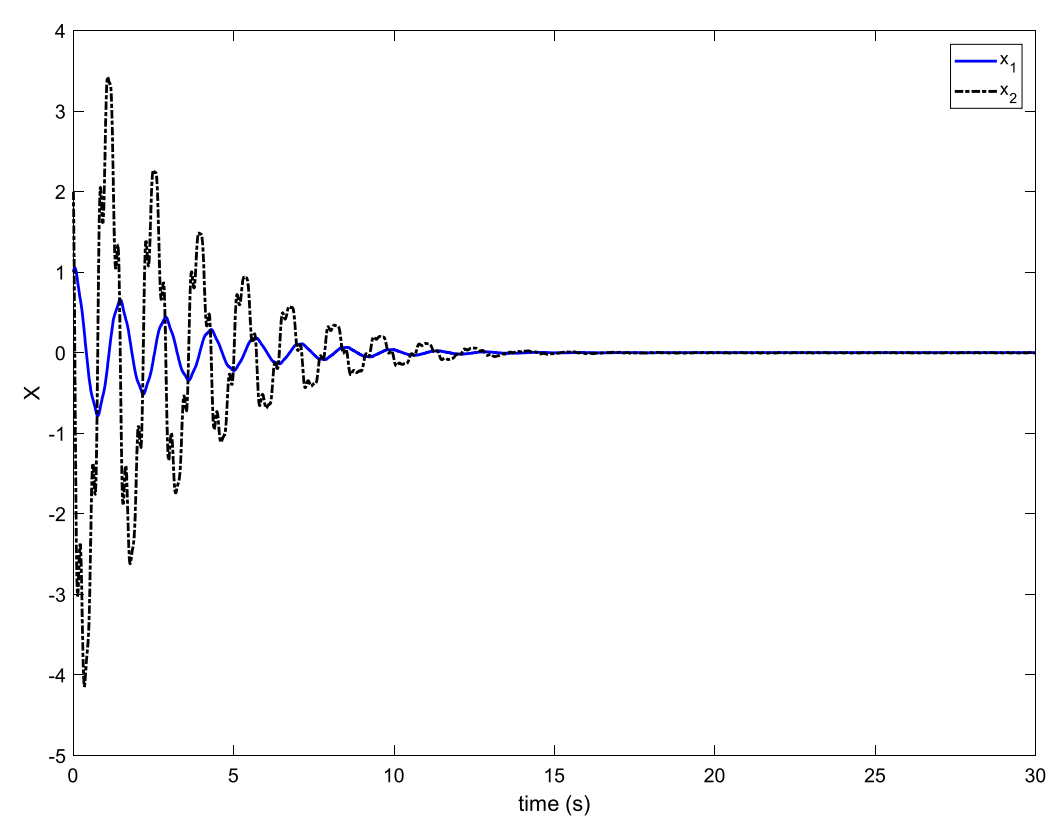

Figure 8 State evolutions of the gyroscope with input nonlinearity

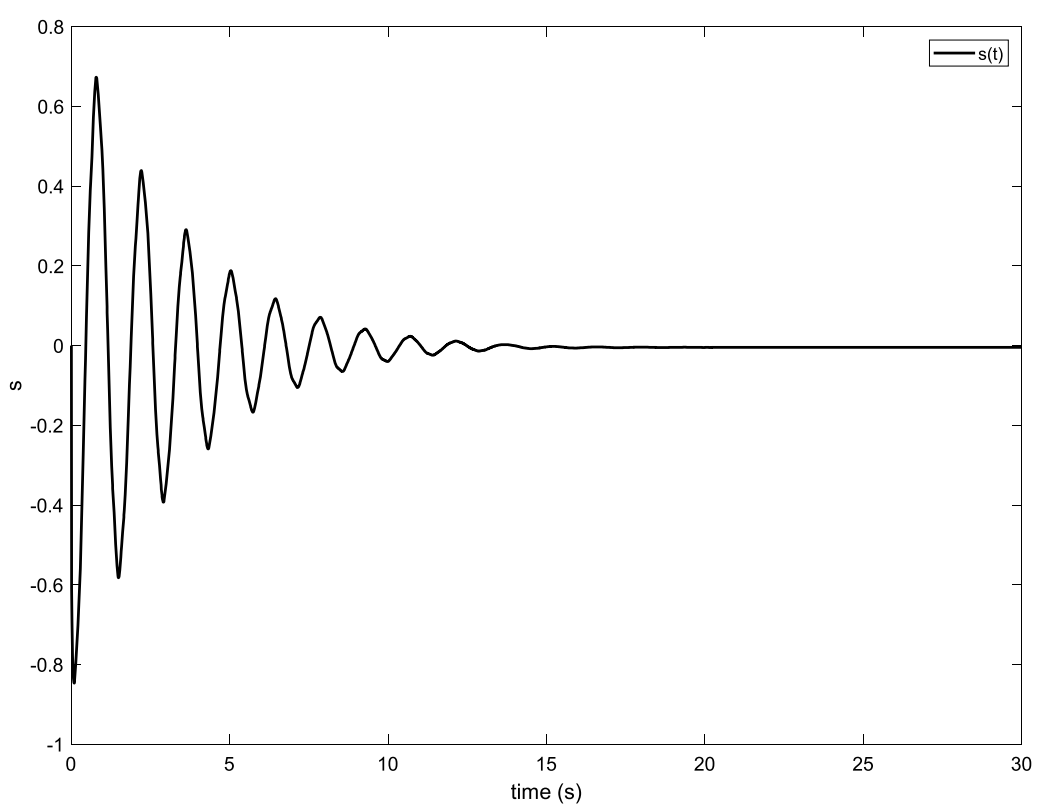

Figure 9 Time evolution of the sliding manifold (44)

Figure 8 shows the states of the controlled gyroscope system in spite of system fluctuations and external disturbances. One observes that the states attain zero after some oscillations. This means that the undesirable chaotic oscillations of the system are indeed attenuated and the effects of the unwanted input nonlinearity as well as control gain uncertainties are fully tackled. The time response of the derived linear sliding surface (44) is plotted in Fig. 9. Clearly, the sliding-mode dynamics is stable with no high frequency 


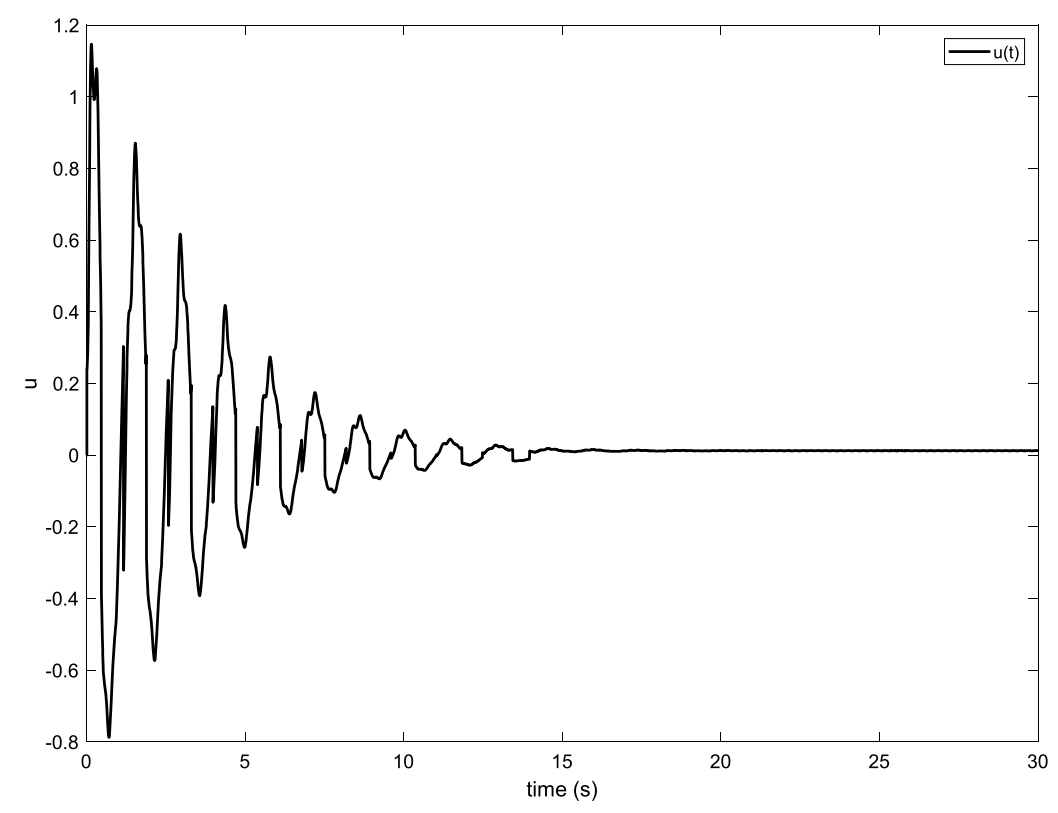

Figure 10 Time evolution of the applied control rule (45)

undesirable oscillations implying that once the oscillations of the original system state trajectories approach this dynamics, they will be converged to the origin. Figure 10 plots the time evolution of the control signal (45) which is disturbed by the sector nonlinearity (43). According to this figure, the control signal involves bounded magnitudes and the convergence to zero is almost achieved. Therefore, the derived robust control technology exhibits a low control energy effort confirming its applicability characteristic in real applications. Also, there is no harmful chattering on the control input depicted in Fig. 10 indicating that the physical actuators are able to implement such smooth control signals. For comparison, the time evolution of the nonlinear input (45) is appeared in Fig. 11. It is seen that the fluctuated control input includes high amplitude oscillations. However, the derived control signal revealed in Fig. 8 is not only with lesser magnitude but also it is smoother than the nonlinear input. Overall simulation results imply that the proposed controller is robust against system uncertainties and external disturbances and can achieve the stabilization goal even if there are unavoidable input nonlinearities and control gain variations on the actuator in physical situations.

\section{Conclusions}

This article proposed the use of fractional-calculus-based control algorithms for oscillation removal of a class of applied integer-order mechanical systems. It was assumed that the system is subjected to input nonlinearities and a robust fractional variable structure switching controller was derived such that guaranteed the oscillation suppression of the uncertain system. Moreover, the case of linear control inputs was handled out using the proposed controller. The introduced control strategy is general and it can be easily modified to handle other actuator nonlinear effects. This article not only proved the stability of the feedback system in the Lyapunov sense, but also analyzed the usefulness and applicability of the designed oscillation suppressor using two illustrative examples. Simulation 


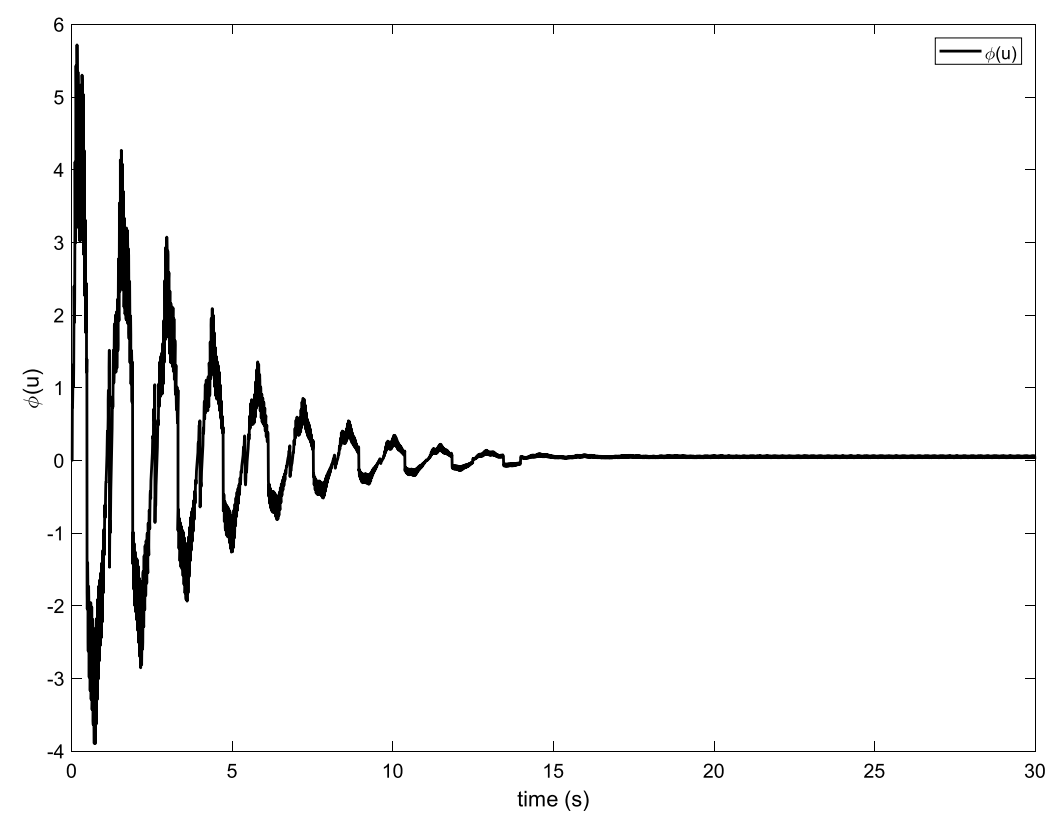

Figure 11 Time evolution of the nonlinear control rule (43)

results indicated that the magnitude of the derived control signal is suitable enough to be realized in practical applications. Further topics along the lines of the present work can be suggested as follows: (i) generalization of the proposed controller for the case of having unknown bounds for the lumped uncertain terms; (ii) introducing a generalized adaptive control scheme for the systems with unknown structural parameters and (iii) applying some modifications to the current controller for overcoming other input nonlinearities such as dead-zones and actuator saturations.

\section{Acknowledgements}

Not applicable.

Funding

This work has received no funding or financial support.

Availability of data and materials

Not applicable.

Ethics approval and consent to participate

Not applicable.

\section{Competing interests}

The author declares that he has no competing interests.

Consent for publication

Not applicable.

\section{Author's contributions}

The whole the research has been done by the single author. Author read and approved the final manuscript.

\section{Authors' information}

Mohammad Pourmahmood Aghababa was born in Tabriz, Iran, in 1983. He received Ph.D. degree in Control Engineering from the University of Tabriz, in 2011. He is a Full Professor at the Urmia University of Technology and has published over 85 international ISI journal papers. His research interests include control theory, fractional calculus and artificial intelligence. Dr. Aghababa is an Associate Editor of the International Journal of Machine Learning and Cybernetics. 


\section{Publisher's Note}

Springer Nature remains neutral with regard to jurisdictional claims in published maps and institutional affiliations.

\section{Received: 11 March 2019 Accepted: 4 September 2019 Published online: 11 September 2019}

\section{References}

1. Karakoc, F., Unal, A., Bereketoglu, H.: Oscillation of a nonlinear impulsive differential equation system with piecewise constant argument. Adv. Differ. Equ. 2018, 99 (2018)

2. Hosham, H.A.: Bifurcations in four-dimensional switched systems. Adv. Differ. Equ. 2018, 388 (2018)

3. Du, C., Xie, L.: Modeling and Control of Vibration in Mechanical Systems. CRC Press, New York (2010)

4. Yan, W., Du, C., Pang, C.K.: Multirate adaptive control of uncertain resonances beyond the Nyquist frequency in high-performance mechatronic systems. Automatica 66, 63-72 (2016)

5. Rajagopal, K., Laarem, G., Karthikeyan, A., Srinivasan, A.: FPGA implementation of adaptive sliding mode control and genetically optimized PID control for fractional-order induction motor system with uncertain load. Adv. Differ. Equ. 2017, $273(2017)$

6. Lam, H.K., Liu, C., Wu, L., Zhao, X.: Polynomial fuzzy-model-based control systems: stability analysis via approximated membership functions considering sector nonlinearity of control input. IEEE Trans. Fuzzy Syst. 23, 2202-2214 (2015)

7. Podlubny, I.: Fractional Differential Equations. Academic Press, New York (1999)

8. Orszulik, R.R., Shan, J.: Output feedback integral control of piezoelectric actuators considering hysteresis. Precis. Eng 47, 90-96 (2017)

9. Cui, R., Zhang, X., Cui, D.: Adaptive sliding-mode attitude control for autonomous underwater vehicles with input nonlinearities. Ocean Eng. 123, 45-54 (2016)

10. He, W., Dong, Y., Sun, C.: Adaptive neural impedance control of a robotic manipulator with input saturation. IEEE Trans. Syst. Man Cybern. Syst. 46, 334-344 (2016)

11. Huang, C.-E., Chen, J.-S.: On the implementation and control of a pneumatic power active lower-limb orthosis. Mechatronics 23, 505-517 (2013)

12. Han, S.I., Lee, J.: Finite-time sliding surface constrained control for a robot manipulator with an unknown deadzone and disturbance. ISA Trans. 65, 307-318 (2016)

13. $\mathrm{Du}, \mathrm{J} ., \mathrm{Hu}, \mathrm{X} ., \mathrm{Sun}, \mathrm{Y}$.: Adaptive robust nonlinear control design for course tracking of ships subject to external disturbances and input saturation. IEEE Trans. Syst. Man Cybern. Syst. (2017). https://doi.org/10.1109/TSMC.2017.2761805

14. Li, G.: Adaptive neural network synchronization for uncertain strick-feedback chaotic systems subject to dead-zone input. Adv. Differ. Equ. 2018, 188 (2018)

15. Zouari, F., Boulkroune, A., lbeas, A.: Neural adaptive quantized output-feedback control-based synchronization of uncertain time-delay incommensurate fractional-order chaotic systems with input nonlinearities. Neurocomputing $237,200-225(2017)$

16. Sun, G., Wu, L., Kuang, Z., Ma, Z., Liu, J.: Practical tracking control of linear motor via fractional-order sliding mode. Automatica 94, 221-235 (2018)

17. Liu, J., Wu, L., Wu, C., Luo, W., Franquelo, L.G.: Event-triggering dissipative control of switched stochastic systems via sliding mode. Automatica 103, 261-273 (2019)

18. Zhao, Y., Wang, J., Yan, F., Shen, Y.: Adaptive sliding mode fault-tolerant control for type-2 fuzzy systems with distributed delays. Inf. Sci. 473, 227-238 (2019)

19. Liu, J., Gao, Y., Su, X., Wack, M., Wu, L.: Disturbance-observer-based control for air management of PEM fuel cell systems via sliding mode technique. IEEE Trans. Control Syst. Technol. 27, 1129-1138 (2019)

20. Liu, J., Yin, Y., Luo, W., Vazquez, S., Franquelo, L.G., Wu, L.: Sliding mode control of a three-phase ac/dc voltage source converter under unknown load conditions: industry applications. IEEE Trans. Syst. Man Cybern. Syst. 48, 1771-1780 (2018)

21. Li, Y., Chen, Y.Q., Podlubny, I.: Mittag-Leffler stability of fractional order nonlinear dynamic systems. Automatica 45 , 1965-1969 (2009)

22. Zhang, F., Li, C.: Stability analysis of fractional differential systems with order lying in (1, 2). Adv. Differ. Equ. 2011 $213485(2011)$

23. Aguila-Camacho, N., Duarte-Mermoud, M.A., Gallegos, J.A.: Lyapunov functions for fractional order systems. Commun. Nonlinear Sci. Numer. Simul. 19, 2951-2957 (2014)

24. Hung, J.Y., Albritton, N.G., Xia, F.: Nonlinear control of a magnetic bearing system. Mechatronics 13, 621-637 (2003)

25. Dadras, S., Momeni, H.R.: Fractional terminal sliding mode control design for a class of dynamical systems with uncertainty. Commun. Nonlinear Sci. Numer. Simul. 17, 367-377 (2012) 